\title{
Externalising Moods and Psychological States to Smooth Pet-robot/Child Interaction through Bluetooth Communication
}

\author{
Ferran Larriba ${ }^{1}$, Cristóbal Raya ${ }^{1}$, Cecilio Angulo ${ }^{1}$, \\ Jordi Albo-Canals ${ }^{2}$, Marta Díaz ${ }^{1}$, and Roger Boldú ${ }^{2}$ \\ 1 GREC Research Group, Universitat Politècnica de Catalunya, \\ Pau Gargallo 5, 08028 Barcelona, Spain \\ flarribagil@gmail.com, \\ \{cristobal.raya, cecilio.angulo, marta.diaz\}@upc.edu \\ 2 La Salle BCN, Universitat Ramon Llull, \\ Quatre Camins, 08022 Barcelona, Spain \\ jalbo@salleurl.edu, rboldu@media.mit.edu
}

\begin{abstract}
Nowadays have already passed more than two years since PATRICIA research project about using pet robots to reduce pain and anxiety in hospitalized children was started and the advances made are more than significant. Patients, parents, nurses, psychologists, engineers... all of them have adopted with illusion Pleo robot, a kind of robotic pet, working hard looking for new procedures and new solutions to the current pediatrics diseases. From this work, a technological contribution is provided going one step beyond to what seems a long path. Concretely, it is wanted to develop a system to wirelessly communicate with Pleo in order to help the coordinator who leads the therapy with the kid, to understand and control Pleo's behaviour at any moment. This article explains how this technological part is being developed and obtained technical results.
\end{abstract}

Keywords: ambient intelligence, bioemotional computing, communication, robotics

\section{Introduction}

How far a robot can help overcome our traumas? Recent advances in the fields of robotics and medicine go further than physical healing. Now to diagnostic robots, to disability robots, to robots for rehabilitation or surgical robots, therapeutic robots are added, designed to motivate and assist the patient with psychological problems. Hence robotherapy consists of the interaction between human patients and robotic creatures (humanoids, pet toys, dummies...) in order to help patients build a positive attitude facing their disease [1].

Since pet robots are mainly considered in this study, we will focus on robots that can be used with a similar therapeutic effect to zootherapy (animal-assisted 
therapy) [2]. Ideally, an animal is the best solution. Unfortunately, these companion animals are not readily available. Concerns of dog bites, allergies, or disease have led to many nursing homes and hospitals to ban this therapy. When this therapy is offered it is a very regulated experience. The animal must be in the company of a trained professional at all times. Additionally, these sessions are scheduled activity and only occur for a few hours or less each day, once or twice a week. As a result of these restrictions this new form of therapy, robotherapy, has emerged.

The Program Child Life was started in 2004 at Hospital Sant Joan de Déu (HSJD), Barcelona, Spain, with the overall aim to design pioneering techniques to improve the children's experience when hospitalized by reducing pain and anxiety [3]. In 2010, more than 200 children and teenagers and their families have participated in this program in HSJD.

The PATRICIA project [4] is based on the use of social robots with the same aim. HSJD cooperates with Universitat Politècnica de Catalunya (UPC), together with La Salle Universitat Ramon Llull (La Salle URL) like technological partners. This kind of projects encourages easily to anyone to get interested in it: it is an innovative project with many unresolved horizons to explore, with a high diverge team, where so different concepts as robotics and human feelings are mixed, therefore multidisciplinary. Engineers, doctors, psychologists... working together looking for improve the life quality of those kids that were unlucky for living together with these disease.

\subsection{Therapeutic Robots}

It is not easy to build a machine able to satisfy the human need for companionship in hard times in the form of pet robots, however companies as Fujitsu, Innvo Labs or PARO Robots are working hard on it. In the last 5 years many studies worldwide have been presented with positive outcomes.

PARO is a baby seal shaped robot designed by Takanori Shibata in Japan in 1993, but did not begin to be commercialized until 2004 [5]. It is equipped with five kinds of sensors: temperature, touch, light, audio and position sensors. Additionally Paro is able to learn behaviors. This pet offers similar benefits as zootherapy, and is used in treatments to people with symptoms of Alzheimer and other disabilities.

Huggable Teddy Bear, is being developed by Fujitsu as a therapeutic companion for hospitals or nursing homes, for health care, education, and social communication applications [6]. Hides a dozen sensors to recognize facial expressions and movements of the patient by the camera on its nose. It is intended to record the patient's emotional state and react accordingly using a range of 300 shares scheduled actions to interact with the people around it.

ROMIBO is an open coded therapeutic robot [7] specially designed to the research and treatment of autism disease in kids. It brings all the wireless connections in order to be remotely controlled.

Finally, Pleo, our chosen platform, is a robot, imitation of a Camarasaurus dinosaur, that exhibits an appealing baby-likeness, expressiveness, and an array 


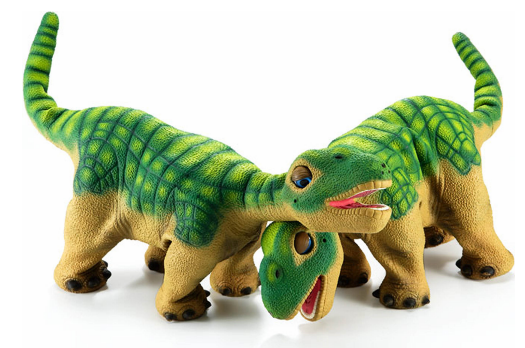

Fig. 1. Pleo the Camarasaurus.

of different behavior and mood modes. Pleo has been tested in several research works [4, 8-10] focused on the effect of Pleo in long-term interaction, especially with children. Another kind of interesting researches with this robot platform is robot ethics [11], which plays a very important role in robotherapy.

Pleo is a commercial entertainment platform developed by Innvo Labs. It is equipped with 2 ARM7 CPUs, 14 motors, 8 touch sensors, IR transceivers, microphones, and 1 camera. It features also a pet like personality which develops in time, internals drives like hunger or sleep, and several mood modes: happy, extremely scared, curious...

The software running in Pleo is divided into three layers: the low-level software deals directly with hardware. Sensor information is provided to the midlevel layer through a blackboard system. The mid-level layer provides the application functional support to the high-level scripting layer. The script layer implements the highest-level functionality of Pleo. This is essentially Pleo's personality, determining how and when he responds to sensor input and internal goals.

\subsection{Robot Autonomy}

Four degrees of Pleo's autonomy can be deployed when interacting with children in the real scenario of the hospital:

1. Full autonomous behavior according to implicit -opaque to users- internal states: Pleo always acts according to its own criteria. The problem is that in this modality, Pleo's behavior is not totally predictable by the user at any time but may be inferred, anticipated or understood by the user according to previous experience in interaction, expectations and social comprehension of Pleos drives and situation awareness.

2. Full autonomous behavior according to observable internal states: The conductor of the intervention can see Pleo's internal states through a graphical interface that externalize or make transparent Pleos's internal states that facilitates the understanding and management of the interaction. 
3. External control of Pleo's states: The coordinator is enabled to modify or control Pleo changing the internal states and letting Pleo perform the correlative activity.

4. External control of Pleo's behaviour: Fully tele-operated control of the movements and actions of Pleo. Always requires the presence of the coordinator to handle the Pleo.

Nowadays the best choice for testing Pleo in the hospital is located between the second and the third levels, hence this will be the approach of this research.

\subsection{Technical Framework}

Latest advances in the research project shows the capability of Pleo's platform helping the kids of HSJD and their families to improve their treatment. Then, the research group presents a prospective view of a new generation of healthcare robots - combining cloud robotics and artificial intelligence - that provide children patients with an effective and individualized assistance [12].

The goal is to supply each young patient with a personal Pleo. Besides, a cloud multi-agent system able to perceive, collect and share hospitalized children status is build, and using artificial intelligence the behaviour of every patient's robot is modified. Finally, as all this information is in the cloud, the system can explore the most effective actions that the Pleo can carry to improve its own patient experience.

Until now, it is not achievable to modify the software system of Pleo due to it is not a full open source. However, it allows to modify some values or to commit some precise actions in a certain moment. For example, it can change or just show how hunger is it, how happy is it, or also to ask it for walking or "to give paw".

Hardware communication with the robot is not so easy as in previous Pleo commercial versions, as long as the new one has the USB port, the serial interface and the Bluetooth connectors behind the battery spot. In order to obtain data in "real time" from Pleo whereas it is powered, it was connected to an external power while stabling a gateway between the USB connector and remote computer.

It is possible to get in real time the distinct values for the different sensors of Pleo adopting the method described. After that, some Bluetooth connections appear connecting a Bluetooth module to the UART port [12] as a bridge between Pleo and a Raspberry Pi. Other processing platforms like Intel Galileo or Edison and wireless communications like ZigBee or WiFi could be also considered, depending on the power, processing or privacy needs. Then, the Raspberry Pi should be set into a little bag specially made for Pleo, and can access to the Internet thanks to a wifi dongle, uploading the data to the cloud. However, there were two problems in that communication: first a lack of space, because original battery of Pleo was employed compacting the wires with the battery and leaving the Bluetooth module in sight ${ }^{3}$ (see Figure 2). It is a trouble taking

\footnotetext{
${ }^{3}$ You can check a video at https://www.youtube.com/watch?v=2qNdZFt8by8.
} 


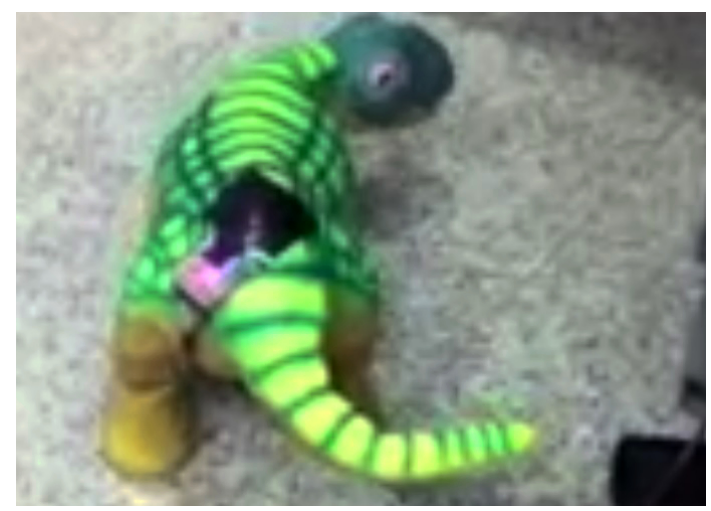

Fig. 2. Pleo's Bluetooth communication [12].

in consideration that Pleo will be used by kids, then it will not accomplish the user specifications. Secondly, new Pleo robots are not equipped with this UART connector, so it could be exclusively tested in old versions.

Therefore, unfortunately each workshop that the psychologist/pediatric group has performed with the children of HSJD (the real scenario) to test the interaction between Pleo and the kids have been performed without any communication provided. And as this platform is new not only for the kids but also for the pediatric group, when Pleo has a bad mood or acts in a non-normal way there is no manner to know exactly what is happening to it. So in that cases the pediatric group interact with Pleo based in their personal experience in order to correct the situation.

According to both, the desired robot autonomy, and the technical drawbacks when using the platform, this set of objectives will be pursued along this work:

- Bluetooth (Bth) communication for Pleo: to build a removable part that will be able to be mounted in any Pleo providing Bth communication.

- Modification of Pleo's states: the coordinator must have the ability to control Pleo to make a specific action because the situation requires it. Android application has been chosen as interface to do that, due to it is an open source easy to spread in the society.

- Bluetooth-battery package: the removable part would fit inside the battery hole, in order to assemble the module in one package.

\section{The Proposed Solution}

This section includes a description of the solution to be developed and other features that have been considered. 

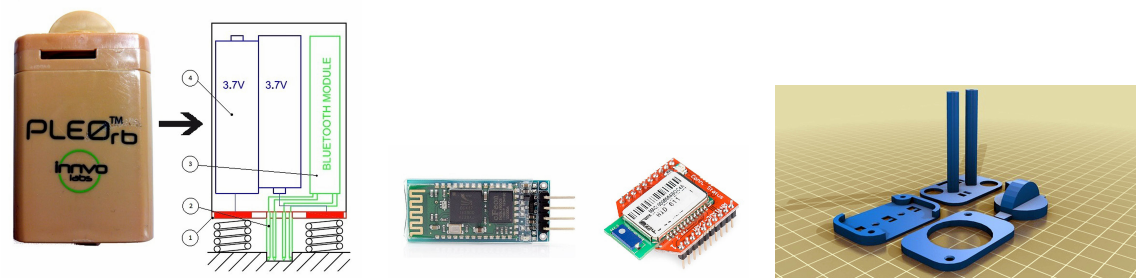

Fig. 3. (Left) Layout of the proposed assembly; (Middle) Modules JY-MCU and RN41; (Right) 3D print model.

\section{$2.1 \quad$ Hardware}

The proposed solution for the hardware challenge, shown in Figure 3, is to switch Pleo's battery for a battery-Bluetooth package. Distinguishable components are listed below.

1. $P C B$ : Its main function is to become the conductive element between the batteries to the springs that feed the robot. Based on the base of the battery, it must fix the 4 pins that establish contact to the Bluetooth output of Pleo.

2. Connector pogo pins: They catch the signal that Pleo sends and will be processed in the Bluetooth module.

3. Bluetooth module: Receives the data signal from the robot and sends it to the device connected. The JY-MCU industrial serial port is one of the cheapest Bluetooth serial port modules in the market, but provided voltage is not enough. Then, a more sophisticated module is needed, for instance RN-41 microchip (see Figure 3(Middle)).

4. Battery pack: Specifications of Pleo's battery are for voltage $(7.4 \mathrm{~V})$, charge $(2800 \mathrm{mAh})$, power $(20.72 \mathrm{Wh})$ and max temperature $\left(60^{\circ} \mathrm{C}\right)$. So, it should be replaced by a couple batteries of $3,7 \mathrm{~V}$ of size AA without problems However, we will check in the Subsection 3.3 how this sentence is not really true.

Once all the components are assembled and tested, a case to pack it with a $3 \mathrm{D}$ printer is made ${ }^{4}$ (see Figure 3 (Right)).

\section{$2.2 \quad$ Interface}

The interface must be as simple and comfortable for the user as possible, using buttons and images to understand Pleo's behaviour. Moreover connecting to the Pleo robot must be user-friendly, Bluetooth must turn 'on' when the app is launched and a button should be present to search Pleo's signal and establish the communication. A first sketch of the main menu is shown in Figure 4. It should have a list of the different Pleo states, be able to modify the emotional

\footnotetext{
${ }^{4}$ In fact, there are already some free models of Pleo's battery case in the Internet to download (http://www.thingiverse.com/thing:31721/\# files
} 


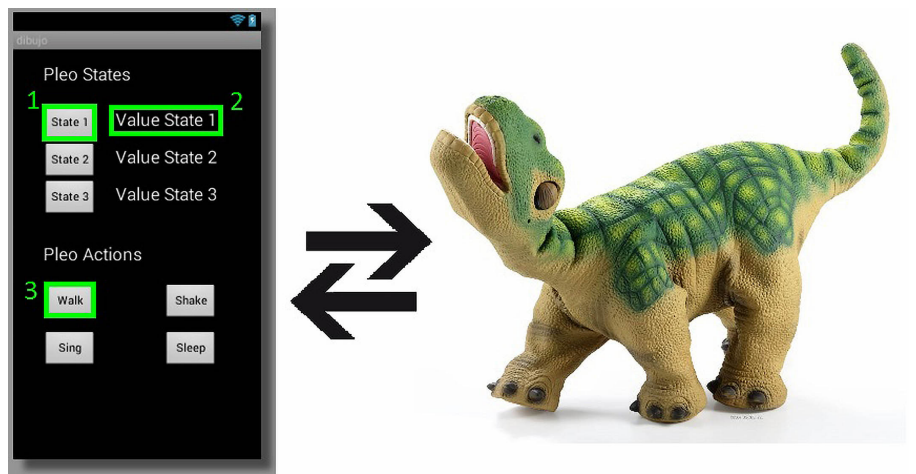

Fig. 4. Sketch of the proposed interface.

status of Pleo, allowing to the user to ask for their value. If needed, it is possible to add more buttons for Pleo Actions, in order to make a specific action when the situation requires it.

\section{Development of the Solution}

In this section, due to lack of space, more that a detailed 'what has been done' description, different troubles and how to manage them will be presented.

\subsection{Connection via Terminal}

Pleo does not support Win 64 bits, so a virtual image for XP 32 bits was implemented. The easiest way to connect to the Pleo is via PuTTY and USB ${ }^{5}$.

\subsection{Bluetooth Assembly}

One of hardest challenges in this work is the difficulty to take measures to build the Bluetooth assembly. On the rear face of the battery inside the hole it is impossible to insert a vernier caliper to measure. Moreover the rear is a little wider than the external perimeter of the hole.

To solve the problem of obtaining the correct pin positions, a thin sheet of transparent plastic have been cut, introduced inside the hole and marked with a fine-tipped pen. Then the plastic is extracted, measured with the vernier caliper and drawn with a CAD tool.

Once drawn the layout, the next step is to build the PCB.

Finally, a 10x1 female header is welded to connect the RN-41 without welding directly to the module, that could damage it.

\footnotetext{
${ }^{5}$ In order to do this, it is necessary to download the Pleo Development Kit, http://ipr10.wikidot.com/pleo
} 


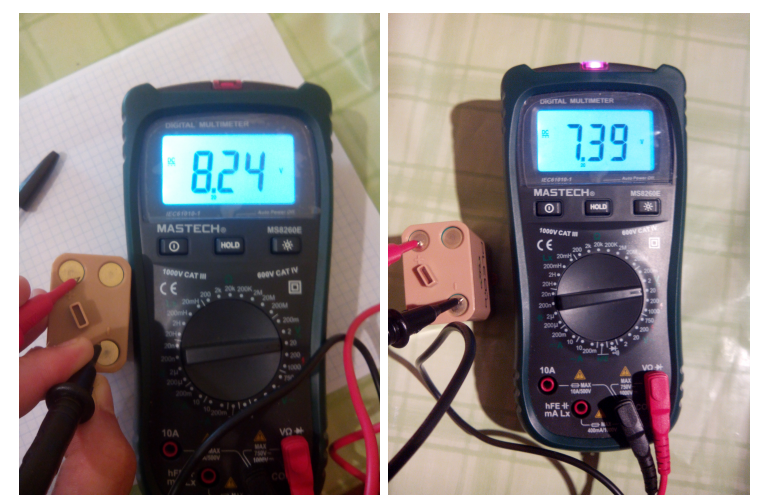

Fig. 5. In the first picture the battery works perfectly. Once Pleo has no power to make it run its battery have exactly $7,39 \mathrm{~V}$.

\subsection{Power Troubles}

When the PCB is set inside the Pleo, batteries power the robot, but after pressing its button to turn it on, the robot does not move. It was thought that probably both batteries are not enough to power the robot and the Bluetooth module, so a button cell of 3,3V was used to power the RN-41, without taking up too much space. Then, after pressing the button, Pleo's makes the sound as wakening up, and sends some data to the screen of the terminal with it is communicating with, but afterwards the robot snores and turns off stopping the communication.

It seemed like a power failure, hence the minimum tension and intensity to make the robot work was measured. There it was discovered that the minimum power to supply to Pleo RB is $7,4 \mathrm{~V}$ (equivalent to $3 \mathrm{~A}$ ), turning the robot to the sleep position in case of reducing its values a little bit. That explains why the same battery that Pleo carries provides more than $8,4 \mathrm{~V}$ when it is completely full.

Another discover was that Pleo RB, unlike USB wired communication, does not allow Bluetooth communication if the robot is not running.

\subsection{Android App}

Figure 6 shows how the interface looks like. First scene is a presentation to introduce the user. Here the application itself turns 'on' the Bluetooth of the smartphone or device where the app is installed.

At the next scene there is a button that user must push once the module RN-41 is powered in order to pair it with the device. Then the app searches the corresponding MAC direction and in case that it does not find it, displays an error message. On the other hand, if the communication is established the app redirects the user to the third scene or command window, where the user sends and receives data from Pleo. 

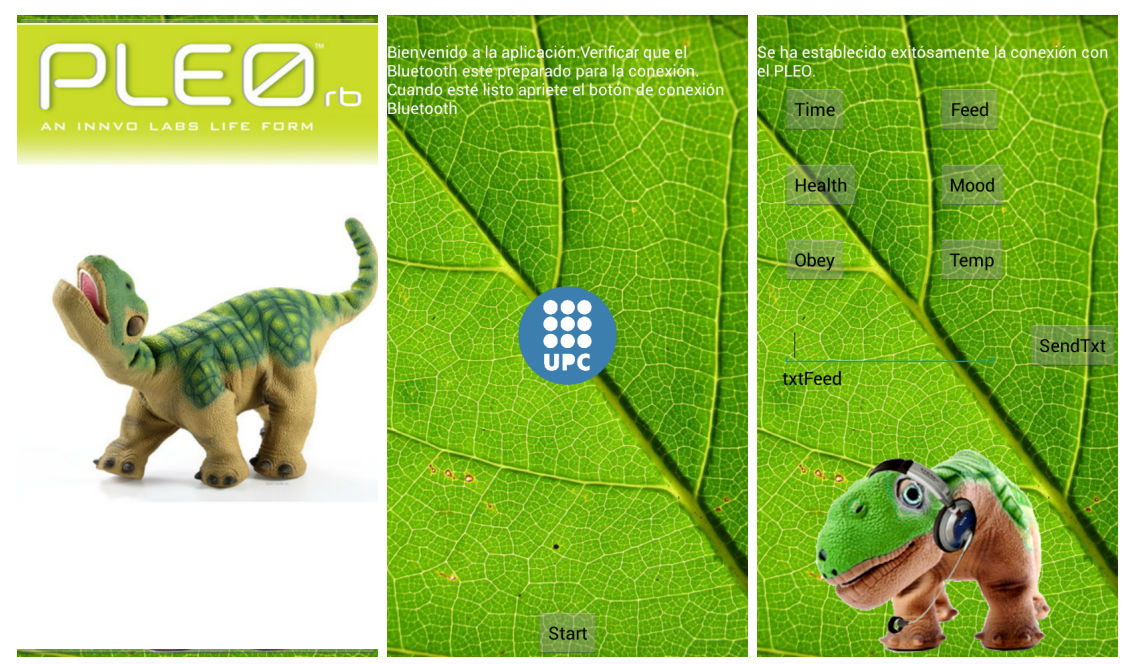

Fig. 6. Different scenes of the application "PleoSays": (Left) Presentation; (Middle) Pairing with Pleo; (Right) Communicate.

When Pleo is switched on, unlike USB communication the robot starts sending data, information of the initialization of the source. Hence, the application developed cannot receive Pleo's states values or to send any motion command until all this initial info has been received and processed ${ }^{6}$.

\section{Results and Discussion}

Wireless communication between Pleo RB and an Android device is finally achieved. With the Android app developed, the user is able to obtain any state of the robot without stopping its interaction with the patient. It also contains an editable textbox to allow the user to send orders to the robot, authorizing the user to a long permission list of actions, as modifying Pleo's states, ordering to commit any action or sound, camera's options, etc.

It has been a success to achieve the communication with the robot connecting the Bluetooth module to the output set behind the battery spot. As newest Pleo's versions have no UART port connection, the incoming works with Pleo should adapt to a solution similar to what has been explained in this article. From now it can be ensured that the bottom connection works properly and can be used in further research.

During the trials, it has been made evident that using a couple of $3,7 \mathrm{~V}$ cells is not enough to make Pleo run due to this values are too close to the operating limit. Using a third cell is needed to make the Pleo robot move.

Moreover, the developed Android app can be installed in many devices and its interface is easily understandable for users.

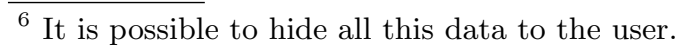


After many hours working with Pleo, several observations can be expressed about it, which three have been highlighted and described below:

- Pleo is not a robust platform. It is a common fact for people that work with Pleo that one day without doing anything different of what they have already done before it does not turn on. Then, why to spend so many effort in working with this tool, if sometimes it breaks without knowing why? The answer is economy. If Pleo "survives" for more than one month working with the kids in therapy, investment can be considered as recovered, since it is very affordable.

- Pleo is selfish. It is not like a dog because Pleo does not react if the kid cries, laughs, plays,... This is a very important issue because it leads to an interest lost by the kid, and is known that the progress of the therapy is directly proportional to the motivation of the patient. If Pleo does not care of the kid, the kid will not care of Pleo and the therapy will be a failure.

- Related to the previous observation, and considering that the future of this research is the cloud net as explained, the cloud should collect the "states" of the kids.

\section{Conclusions}

The first one of the objectives of this project, the Bluetooth communication with Pleo, has been achieved completely. Using the PCB with the extensible pins, we can supply Bluetooth communication to any Pleo, even those without UART connection.

The second objective, to modify Pleo's states from an Android device, or any of their possible actions, has been successfully achieved too, so helping the coordinator to understand and to control the robot using an easy app.

The third objective, to assemble a Bluetooth + Batteries package has not been fully completed. Pleo will work using 3 (not 2) AA cells and it will fit to the designed package in this work, but it was not be completely developed, since $3 \mathrm{D}$ cage was build for two AA cells. However, it should be possible to build this assembly.

As far as Pleo is a commercial closed platform, alternative platforms should be considered in the near future. In this sense, two technical research lines are opened. The first one is either to consider commercial platforms as Pleo's alternative or to develop a pilot robotic platform to be certified for commercial use. The second line is working in the possibility to certify the employed device as a medical device for therapeutic purposes.

Acknowledgments. This research was supported in part by the PATRICIA Research Project (TIN2012-38416-C03-01,02,03), funded by the Spanish Ministry of Economy and Competitiveness. Thanks to Hospital Sant Joan de Déu, the team of nurses and volunteer corps by shared experience. 


\section{References}

1. Libin, E., Libin, A.: New diagnostic tool for robotic psychology and robotherapy studies. Cyberpsy., Behavior, and Soc. Networking 6(4), 369-374 (2003)

2. López-Cepero, J., Rodríguez-Franco, L., Perea-Mediavilla, M.A., Blanco Piñero, N., Tejada Roldán, A., Blanco-Picabia, A.: Animal-assisted Interventions: Review of Current Status and Future Challenges. Int. J. Psych. and Psycholog. Therapy 14(1), 85-101 (2014)

3. Serrallonga-Tintoré, N., Cabré-Segarra, V.: El cuidado emocional en la prevención del dolor posquirúrgico en niños y adolescentes. Rev. Psicopat. Salud Mental Niño y Adol. 16, 49-56, (2010)

4. Angulo, C., Garriga, C., Luaces, C., Pérez, J., Albo-Canals, J., Díaz, M.: Pain and Anxiety Treatment based on Social Robot Interaction with Children to improve Patient Experience. Ongoing Research. In: Proceedings of the XIV ARCA days. Qualitative Systems and its Applications in Diagnose, Robotics and Ambient intelligence, pp. 25-32. Salou, Spain (2012)

5. Taggart, W., Turkle, S., Kidd, C.D.: An Interactive Robot in a Nursing Home: Preliminary Remarks. In: Toward Social Mechanisms of Android Science. Cognitive Science Society, Stresa, Italy (2005)

6. Stiehl, W.D., Lieberman, J., Breazeal, C., Basel. L., Lalla, L., Wolf, M.: The Design of the Huggable: A Therapeutic Robotic Companion for Relational, Affective Touch. In: Proceedings of AAAI Fall Symposium on Caring Machines. Washington D.C. $(2005)$

7. Shick A.: Romibo robot project: an open-source effort to develop a low-cost sensory adaptable robot for special needs therapy and education. In: Special Interest Group on Computer Graphics and Interactive Techniques, ACM SIGGRAPH 2013 Studio Talks. Anaheim, California (2013)

8. Díaz, M., Andrés, A., Angulo. C.: Robots sociales en la escuela. Explorando la conducta interactiva con niñ@s en edad escolar. In: ROBOT 2011 : robótica experimental, pp. 622-625. Sevilla, Spain (2011)

9. Chung-Chang, C.Y., Díaz, M., Angulo, C.: The Impact of Introducing Therapeutic Robots in Hospital's Organization. In: Bravo, J., Hervás, R., Rodríguez, M. (eds.) IWAAL 2012. LNCS, vol. 7657, pp. 312-315. Springer, Heidelberg (2012)

10. Heerink, M., Díaz, M., Albo-Canals, J., Angulo, C., Barco, A., Casacuberta, J., Garriga, C.: A field study with primary school children on perception of social presence and interactive behavior with a pet robot. In: 21st IEEE International Symposium on Robot and Human Interactive Communication (Ro-Man 2012), pp. 1045-1050. IEEE Press, New York (2011)

11. Darling, K.: Extending Legal Rights to Social Robots. In: We Robot Conference. University of Miami (2012)

12. Navarro, J., Sancho, A., Angulo, C., Garriga, C., Ortiz, J., Raya, C., Miralles, D., Albo-Canals, J.: A Cloud Robotics Architecture to Foster Individual Child Partnership in Medical Facilities. Workshop on Cloud Robotics, RSJ IEEE/RSJ International Conference on Intelligent Robots and Systems (IROS 2013). Tokyo Big Sight, Japan (2013) 